\title{
Application of Artificial Intelligence in the Field of Forensic Medicine
}

\author{
Manoj Pathak ${ }^{1}$, Himanshi Narang ${ }^{2}$ \\ ${ }^{1}$ Professor, Department of Forensic Medicine and Toxicology, All India Institute of Medical Sciences, Patna,

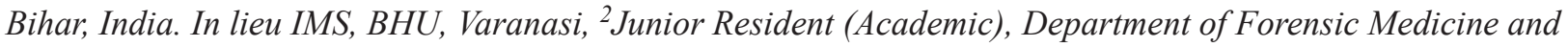 \\ Toxicology, All India Institute of Medical Sciences, Patna, Bihar, India
}

\begin{abstract}
Artificial Intelligence (AI) is a branch of software engineering that is basically concerned with the creation of keen machines which fill in as humans. The blend of master and machine is needed to diminish the functioning load and understand more cases. AI has gotten vital for practically all zones including software engineering, security designing, criminal science, brain research, and advanced mechanics. AI, neural organization and profound learning are unified to one another and they all go under the roof of computerized reasoning. $\mathrm{AI}$ is a piece of man-made brainpower which permits a machine or software program to gain from authentic exercises or patterns to foresee new yield esteems by utilizing explicit calculations. The stakes are unimaginably high; "shrewd" calculations remain to assume a basic part in choices to automatically submit or sedate people, the arrangement of condemning suggestions, and surprisingly the direction of focused police surveillance. The values frameworks that guide these algorithms, for better or worse, will be controlled by those that design them.
\end{abstract}

Keywords: Artificial Intelligence, Risk Assessment, Machine Learning, Forensic Psychiatry, Violence Risk, AI, Neuroprediction

\section{Introduction}

Artificial Intelligence (AI) is a branch of software engineering that is basically concerned with the creation of keen machines which fill in as humans. This includes the development of machines ready to take part in humanlike thought processes such as learning, reasoning, adapting, self-correction, etc. and act rationally. ${ }^{(1)}$ Utilization of PC innovation to animate smart conduct and basic intuition practically identical to people. The improvement of significant learning estimations has acquired superb ground in the latest years. ${ }^{(2)}$ Searching

\section{Corresponding author:}

Himanshi Narang, Junior Resident (Academic), Department of Forensic Medicine and Toxicology, All India Institute of Medical Sciences, Patna, Bihar, India. +91-8168089511, himanshin07@gmail.com the evidence from an information base feeder with the target should be learned by support learning. The savvy criminal examination, precise scientific assessment and fair legal framework are the mount of progress for criminal equity. The programmed Add-In customized with a calculation pointing and expect the Machine through experimentation to accomplish that objective endeavouring to move over the most article recognition until it finds with various percentages. ${ }^{(3),(4)}$

The blend of master and machine is needed to diminish the functioning load and understand more cases. Clearly, one requirements to support the results and quality confirmation is for the most part significant (2) AI has gotten vital for practically all zones including software engineering, security designing, criminal science, brain research, and advanced mechanics. Particularly, Profound Learning, enlivened by the construction and capacity of the cerebrum, has been the 
Medico-legal Update, October-December 2021, Vol.21, No. $4 \quad 57$

significant forward leap in the AI field and it has enacted the AI concentrate in different fields. ${ }^{(2)}$

The previous studies give three ramifications to stakeholders in AI fields. To begin with, because of the double use nature of AI, researchers and engineers ought to see that AI technique might be utilized to perpetrate criminal offenses, despite the fact that the strategy is intended for genuine use.

Since AI is a double-edged sword, stakeholders in AI field need exact proficient morals. Second, entirely unexpected sorts of safety dangers, that have not been considered up until this point, will arise. As AI can finish tasks that have been viewed as difficult to be prepared by individuals or customary projects, the dangers will be outside the essential extent of known dangers; AI researchers ought to team up intimately with experts in assorted fields to forestall the security dangers of computer-based intelligence and react to AI wrongdoing. At last, AI security territory ought to gain from preliminaries and blunders of network safety region. As depicted in past investigations, the predictable artificial intelligence violations are firmly associated with cybercrime. The cybercrime originated from the dual-use nature of ICT; the current situation of AI security resembles the initial phase of cybersecurity. (5) Expanding number of talented assets is one alternative to accelerate examination measure, while other choice is to make the instruments sufficiently brilliant to turn out keenly for investigating dubious information. ${ }^{(6)}$

However, in an event for solving a instance of murder or tracking the culprits of sexual abuse or searching evidences for record misinterpretation requires a hounded police work. ${ }^{(7)}$ A solitary finger impression may require a lot of suspected finger impression information to look at and physically this is likewise exceptionally monotonous and tedious task. ${ }^{(8)}$ Digital forensics is a profoundly propelling field and requires the more clever dissecting strategies for tackling its unpredictable issues by investigation of a gigantic measure of information sets. $^{(9)}$

Even assessment or correlation of tremendous volumes of potentially important confirmations like pictures, recordings, sound, fingerprints, penmanship styles, and so on in a precise and convenient way is a meticulous, tedious assignment with conceivable human blunders because of ineptitude or exhaustion or different variables. ${ }^{(10)}$

Consequently, assessment of information utilizing ordinary serious and manual methodologies are not equipped for managing issues and entanglements at present found in forensic science. The human expert or investigator or practitioner can be drained, one-sided, biased, clumsy or passionate during the assessment process. This often lead to increasing errors or bogus results which lead to failure of investigation and premature delivery of justice. ${ }^{(10),(11)}$ Imagine a scenario where all of these errands are performed by a software programme that acts shrewdly? ${ }^{(2),(4)}$

\section{What's New?}

AI, neural organization and profound learning are unified to one another and they all go under the roof of computerized reasoning. AI is a piece of man-made brainpower which permits a machine or software program to gain from authentic exercises or patterns to foresee new yield esteems by utilizing explicit calculations. AI frameworks can gain more from progressive cases and can be presented to different cases in practically no time. Fundamentally, this modern revolution is distinguished from previous ones by the sort of assignments being automated. When algorithms utilize machine learning, new information are continually consolidated to improve and refine a prescient model. ${ }^{(12)}$

\section{Forensic Odontology}

There are such advanced calculations of AI which are intended for individual distinguishing proof through dental findings during mass disaster investigations. ${ }^{(13)}$ can likewise be prepared to assess the sex of a person from skeletal remains. AI calculations dependent on 
neural organizations require no critical mastery to carry out. They are exact, fast to utilize and have a capacity to dispose of human predisposition from sex assessment of skeletal remains. Neural networks can possibly democratize measurable humanities and effect fields going from antiquarianism to forensic science. ${ }^{(14)}$

\section{DNA Evidence}

DNA analysis software was first developed a decade ago. DNA evidence often isn't as watertight as people suspect. Delicate procedures created in the course of recent years imply that police would now be able to distinguish minute hints of DNA at a crime location or on a piece of proof. Yet, traces from a perpetrator are frequently blended in with those from numerous others that have been moved to the crime site, for instance by means of a handshake.

Researchers have created algorithms to isolate this DNA soup and to gauge the overall measures of every individual's DNA in a sample. This "probabilistic genotyping" techniques have empowered scientific agents to demonstrate how likely it is that a person's DNA was remembered for a blended sample found at the crime location. Also, presently, more modern AI strategies are being created trying to separate DNA profiles and attempt to work out whether a DNA test came straightforwardly from somebody who was at the crime location, or whether it had recently been guiltlessly moved.

Evidence derived from DNA mixture software very quickly ran into challenges from defence teams (including that of OJ Simpson), ${ }^{(15)}$ worried that the indictment ought to exhibit that the product was effectively validated. How accurate were the outcomes, and what was the known error rate? How precisely did the product function and could it oblige safeguard speculations? Were the outcomes actually so trustworthy that a jury could securely convict?

It is a major precept of the law that proof should be available to investigation. The jury can't depend on uncovered declarations (claims made without proof), regardless of who makes them and what ability they have. Be that as it may, the proprietors of the product contended it was their secured protected innovation and how it functioned shouldn't be disclosed. However, the product hasn't totally addressed the issues of DNA blends and little, degraded samples. This is muddled by the way that various individuals shed DNA at various rates - a wonder known as their "shedder status". Add the way that DNA is moved at various rates across various surfaces and in various ecological conditions and it might turn out to be practically difficult to know precisely where DNA in an example came from. This issue of "move and steadiness" takes steps to truly sabotage criminological DNA. ${ }^{(16)}$

\section{POST-MORTEM INTERVAL}

At whatever point, investigator encounters a deceased, then to find out the time between the death and discovery of death body, is the primary task. Post mortem interval is the time since death. This PMI and forensic report will help the court, regardless of whether to acknowledge or dismiss the assertions of suspects and witnesses. ${ }^{(17)}$ 


\section{Prediction Of Time Of Death}

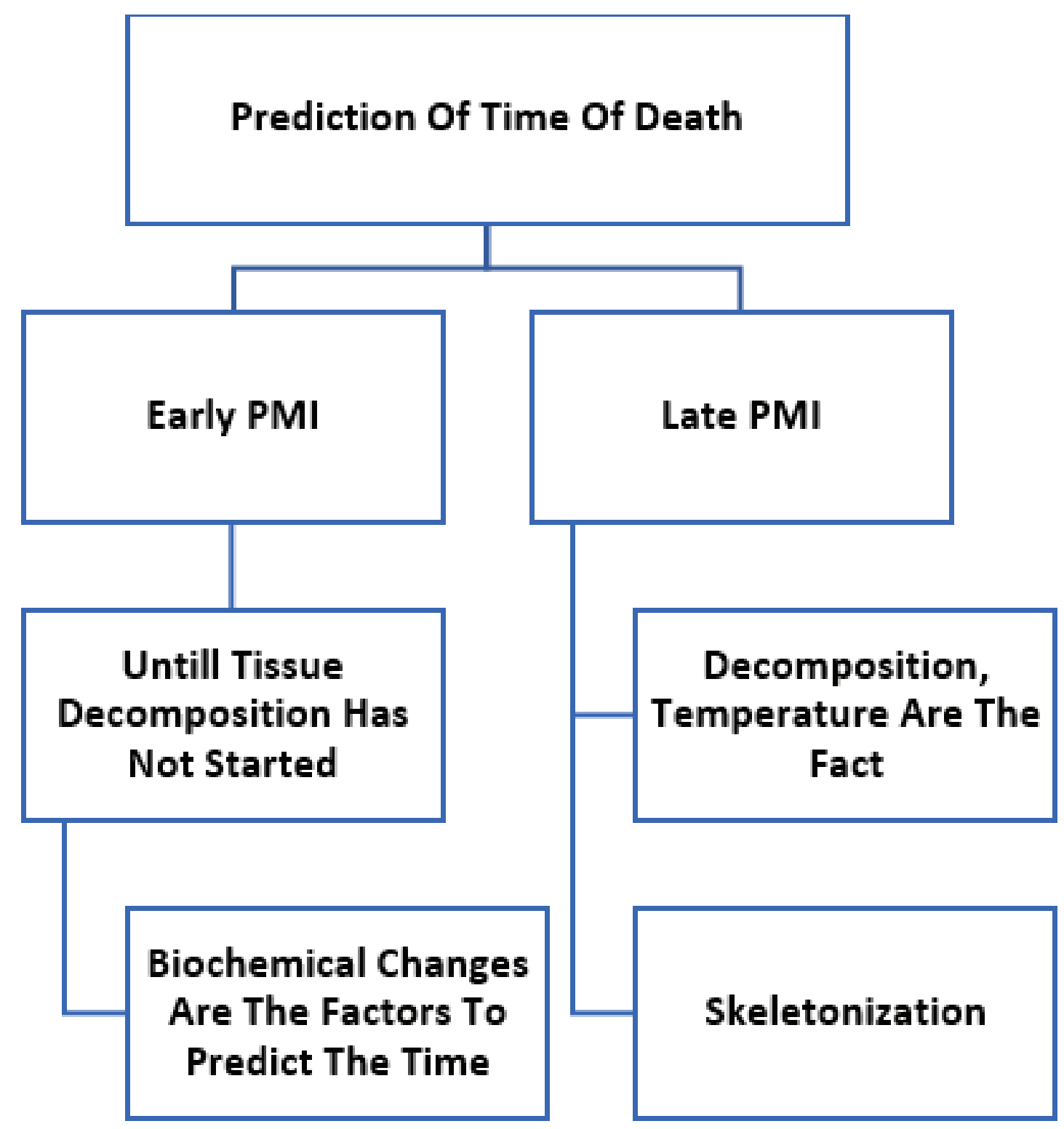

Flowchart 1: Showing prediction of time since death considering early PMI and late PMI.

The idea of assessing the time since death during forensic examination depends on the utilisation of AI device estimating markers in blood such as LDH and $\mathrm{AST}$ as protein and cholesterols as lipid, as well as $\mathrm{pH}$ of blood. When the profile is given to the gadget, it will examine the information and gives us the right outcome with the prediction of time of death. 


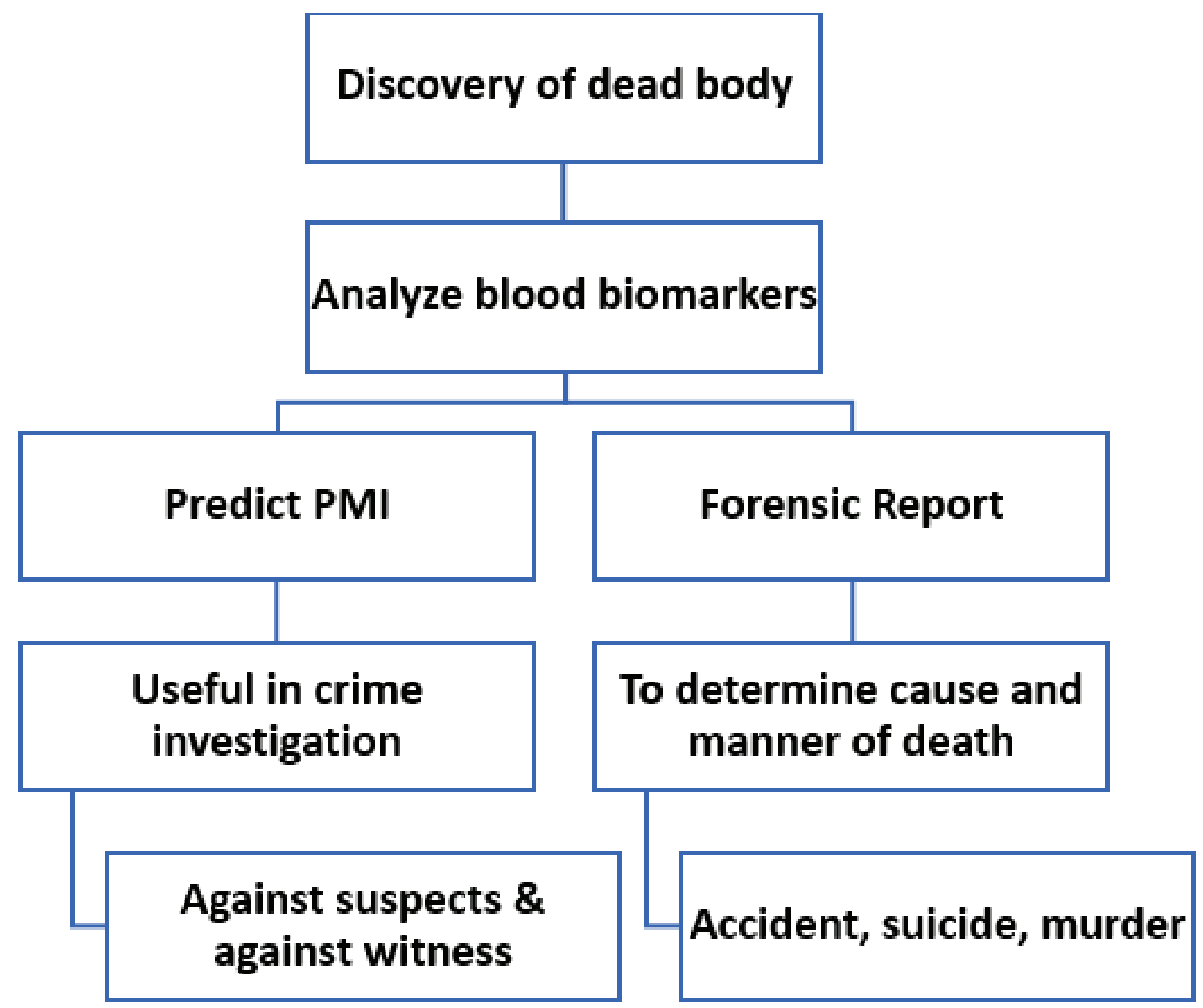

Flowchart 2: Showing application of machine learning to predict post-mortem interval.

Data processing is the principle key factor utilized for the understanding of information and examination with the data set to assess the PMI. Providing profile to the AI machine and getting the ideal yield is the solitary interaction. Practicality of such gadgets ought to be assessed and choice made with respect to utilizing this gadget. ${ }^{(18)}$

\section{Ballistics}

Counterfeit Neural Organization can control specialists for looking through explosive, cartridge case and help them for correlation of projectile imprints, gun ID and other ballistic proof from the information base itself with the assistance of picture handling with no manual obstruction. ${ }^{(19),(20)}$
THREE-DIMENSIONAL

FACIAL RECONSTRUCTION FROM UNIDENTIFIED SKULL

The framework depends on a data set of CT head scans, which as far as anyone is concerned is the lone such data set of this extent in presence. ${ }^{(21)}$ The capacity to produce an individual's particular face space empowers the client to consolidate earlier information like assessment old enough and weight straightforwardly into the reconstruction. ${ }^{(22)}$

\section{NEUROPREDICTION AND FORENSIC PSYCHIATRY}

Risk evaluation is a vital part of the criminal equity framework. Lately, there has been a developing interest 
in the advancement of new devices and strategies to improve risk assessment in the field of forensic psychiatry and criminal justice. ${ }^{(23)}$ The focal point of these techniques is to distinguish high-risk and low-risk offenders correctly. Contingent upon the purview, they are utilized to advise a reach regarding medico-legal choices, for example with respect to condemning, parole, common responsibility, capital punishment, disposition in juvenile courts, and discharge following findings of insanity. ${ }^{(24)}$

In recent years, A.I. is being utilized to improve the prescient accuracy of risk assessment. The utilization of calculative risk assessment has developed alongside the exploration in the field of neuroimaging, prompting the advancement of 'brain-reading' strategies that are, to some restricted degree, ready to interpret mental states dependent on an individual's cerebrum action, ${ }^{(25)}$ or to order individuals in bunches dependent on their brain design and function. ${ }^{(26)}$ A potential legal utilization of the procedure is to distinguish dangerous offenders. furthermore, neuroimaging has prompted the advancement of what can be called 'A.I. Neuroprediction,' which is the utilization of primary or functional brain parameters combined with AI strategies to make clinical or behavioural predictions.

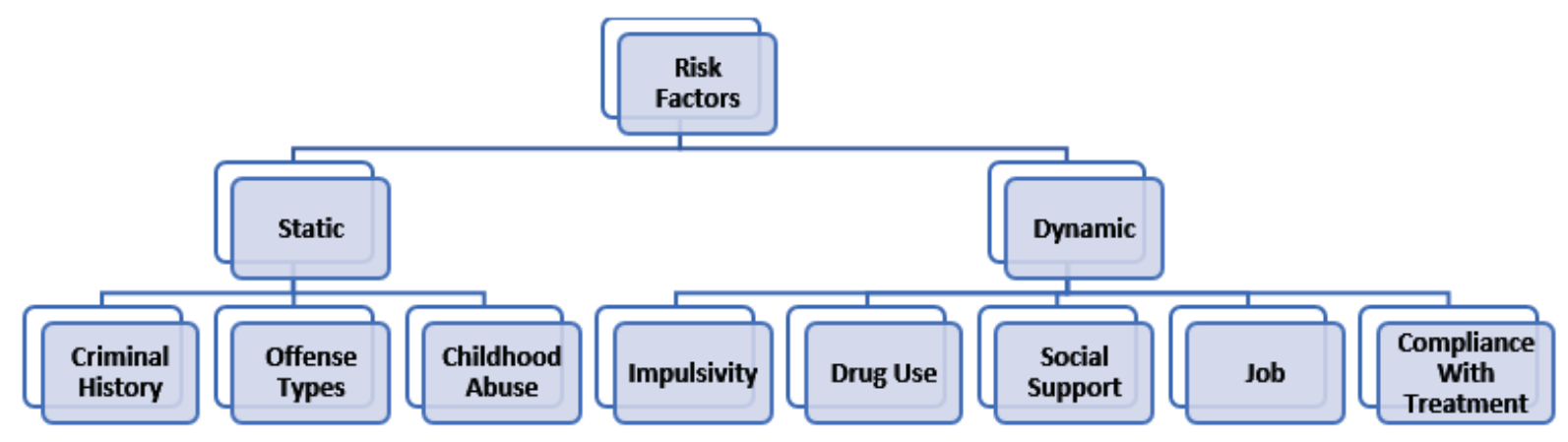

Flowchart 3 showing risk factors associated with forensic psychiatry.

As of now, the consequences of risk assessment tools, notwithstanding, are a long way from great, particularly for long haul expectation; current criminal risk evaluation tools show poor to moderate accuracy, and a decent harmony between bogus positives and bogus negatives is an issue that ought to be thought of, depending both on the social and political setting and on the phase of the criminal equity measure in which the tool is utilized. ${ }^{(27)}$ By and large, when a risk assessment tool identifies a person as low-risk, it is often right. Nonetheless, if the tool identifies someone as high risk, this is regularly erroneous, and practically the greater part of people focused as high-hazard are inaccurately classified. ${ }^{(28)}$ The utilization of AI based frameworks in criminal equity and forensic psychiatry ought to be exposed to generous guideline to shield residents from framework blunders or misuse. ${ }^{(29)}$

\section{VIOLENCE RISK ASSESSMENT}

Dependable and precise appraisal of savagery risk stays a subtle objective for forensic psychiatrists. regardless of whether in the trauma centre or in the court, violence risk assessment remains to a great extent the space of well-qualified assessment. ${ }^{(28)}$ Existing violence risk assessment tools, which apply a fixed arrangement of hazard components to a populace, are unequipped for representing this intricacy. Calculations driven by profound learning are dynamic. ${ }^{(12)}$ This benefit turns out to be considerably more articulated when concentrating particularly uncommon occasions, e.g., spree killings. 
Medico-legal Update, October-December 2021, Vol.21, No. 4

$\mathrm{AI}$ is additionally coming to violence risk assessment on inpatient wards. A report from Netherlands applied AI techniques to a large data set of clinical notes to anticipate future fierce conduct among mental inpatients; ${ }^{(30)}$ the outcomes showed commonly equivalent or predominant prescient when compared with existing tools.

Facebook has unobtrusively carried out its AI driven suicide prevention tool, which analyses huge abundance of client information to assess suicide risk. (31) In the coming years, forensic psychiatrists will be progressively approached to decipher information from shrewd calculations in making proposals including competency, responsibility, condemning choices, the utilization of involuntary drugs, and more. ${ }^{(12)}$

\section{DISABILITY CERTIFICATION}

"AI can be a game changer for people with disabilities. Already we're witnessing this as people with disabilities expand their use of computers to hear, see and reason with impressive accuracy,"

\section{-Brad Smith, President and CLO at Microsoft.}

The world populace is more than 7 billion and over $15 \%$ of the populace is genuinely tested in some structure. As per studies, just one of every ten individuals with a type of disability approach any type of assistive innovations or items. Braille for the visually impaired, hearing aid for the hard of hearing, wheelchairs for a physical malady, are among the most widely recognized aid that human intelligence has accompanied up. ${ }_{(32)}$

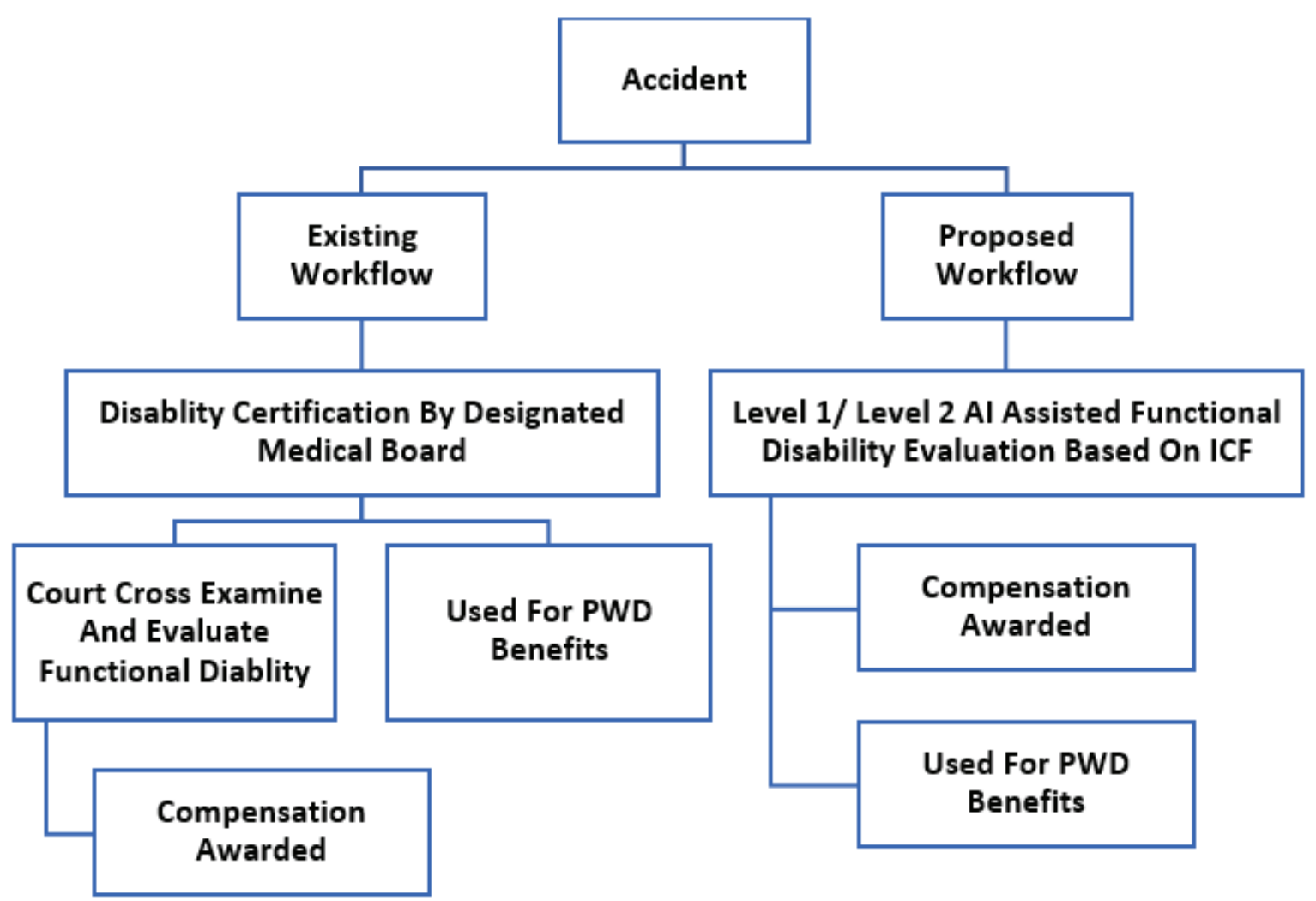

Flowchart 4 showing existing workflow and proposed workflow for disability certification using artificial intelligence. 
The "International Classification of Functioning, Disability and Health"(33) code makes it simpler for AI systems to program and train systems. Judiciary will profit by using this objective classification. AI can help the extraordinarily abled. Advances in AI like Speech-to-Text transcription, predictive text, and facial acknowledgment guarantee a more comprehensive future for the entirety of mankind.

\section{Conclusion}

AI utilizes numerical calculations to finish undertakings like coordinating with a look to a specific arrangement of feelings. It is a need for the hour. Be that as it may, urgently, it can learn through a cycle of experimentation and bit by bit controls its hidden calculations to turn out to be more proficient. It's this interaction of control and change that isn't generally straightforward. ${ }^{(34)}$ The software rolls out its improvements staggeringly quickly as per its own garbled rationale. It can determine fabulously effective outcomes yet we can't say how it did as such. It acts like a black box that takes sources of info and gives outcomes, however whose internal functions are imperceptible. ${ }^{(5)}$

Programmers can go through a clearer improvement process but it is slower and less efficient. This transparency issue influences numerous more extensive utilizations of AI. However, in the event that this innovation is effective, it could present another issue, since it's as of now difficult to see precisely how AI arrives at its decisions. ${ }^{\left({ }^{35)}\right.}$ What's more, how might we believe innovation to give fundamental proof in the event that we can't cross examine how, it delivered that proof in any case?

It can possibly open the way to considerably more miscarriages of justice and this absence of transparency might be a boundary to the innovation's utilization in legal examinations ${ }^{(34)}$ The stakes are unimaginably high; "shrewd" calculations remain to assume a basic part in choices to automatically submit or sedate people, the arrangement of condemning suggestions, and surprisingly the direction of focused police surveillance. The values frameworks that guide these algorithms, for better or worse, will be controlled by those that design them. ${ }^{(36)}$

\section{Ethical Clearance - Yes}

Conflict of Interest- The authors have no competing conflict of interest.

\section{Source of Funding - Nil}

\section{References}

1. Kok JN. Artificial Intelligence: Definition, Trends, Techniques and Cases. Artif Intell. :5.

2. Geradts Z. Forensic Psychology \& Criminology 2019: Artificial Intelligence, deep learning in forensic science- Zeno Geradts- Forensic Institute of the Ministry of Security and Justice. : 1 .

3. Hassan S. Implement Artificial Intelligence AddIn to execute Digital Forensics Investigation Software. : 1 .

4. Jadhav EB, Sankhla MS, Kumar R. Artificial Intelligence: Advancing Automation in Forensic Science \& Criminal Investigation. 2020;15(1533): 13 .

5. Jeong D. Artificial Intelligence Security Threat, Crime, and Forensics: Taxonomy and Open Issues. IEEE Access. 2020; 8:184560-74.

6. Rughani PH. Artificial Intelligence Based Digital Forensics Framework. Int J Adv Res Comput Sci. 2017 Oct 20;8(8):10-4.

7. Baraniuk $\mathrm{C}$. The new weapon in the fight against crime - BBC Future [Internet]. Machine Minds. 2019 [cited 2021 Apr 22]. Available from: https:// www.bbc.com/future/article/20190228-how-ai-ishelping-to-fight-crime

8. Moses KR, Higgins P, Probhakar S, Swann S, McCabe M. AutomAted Fingerprint identiFicAtion system (AFis). National Institute of Justice, Washington DC, 6-1.; 2011.

9. Mitchell F. The use of Artificial Intelligence in digital forensics: An introduction. Digit Evid Electron Signat Law Rev [Internet]. 2014 Jan 27 [cited 2021 Apr 6];7(0). Available from: http:// journals.sas.ac.uk/deeslr/article/view/1922

10. Green M. Human Factors In Forensic Evidence [Internet]. [cited 2021 Apr 22]. Available from: https://www.visualexpert.com/Resources/ forensics.html 
64 Medico-legal Update, October-December 2021, Vol.21, No. 4

11. Turvey BE. Criminal Profiling: Evidence, Experts, and Miscarriages of Justice. In: Koen WJ, Bowers $\mathrm{CM}$, editors. The Psychology and Sociology of Wrongful Convictions [Internet]. San Diego: Academic Press; 2018 [cited 2021 Apr 22]. p. 1-43. Available from: https://www.sciencedirect. com/science/article/pii/B9780128026557000010

12. Cockerill RG. Ethics Implications of the Use of Artificial Intelligence in Violence Risk Assessment. J Am Acad Psychiatry Law. 2020;48(3):5.

13. Takano H. Personal Identification From Dental Findings Using AI And Image Analysis Against Great Disaster In Japan. J Forensic Leg Investig Sci. 2019 Dec 31;5(3):1-4.

14. Bewes J, Low A, Morphett A, Pate FD, Henneberg M. Artificial intelligence for sex determination of skeletal remains: Application of a deep learning artificial neural network to human skulls. J Forensic Leg Med. 2019 Feb;62:40-3.

15. O. J. Simpson murder case. In: Wikipedia [Internet]. 2021 [cited 2021 Apr 22]. Available from: https:// en.wikipedia.org/w/index.php?title=O__J._ Simpson_murder_case\&oldid $=1019018939$

16. Richmond K. AI could revolutionise DNA evidence - but right now we can't trust the machines [Internet]. The Conversation. [cited 2021 Apr 10]. Available from: http://theconversation.com/aicould-revolutionise-dna-evidence-but-right-nowwe-cant-trust-the-machines-129927

17. Cockle DL, Bell LS. Human decomposition and the reliability of a 'Universal' model for post mortem interval estimations. Forensic Sci Int. 2015;253(August):136.e1-136.e9.

18. Mesejo P, Martos R, Ibáñez Ó, Novo J, Ortega M. A Survey on Artificial Intelligence Techniques for Biomedical Image Analysis in Skeleton-Based Forensic Human Identification. Appl Sci. 2020 Jul 8;10(14):4703.

19. Bobbili R, Ramakrishna B, Madhu V. An artificial intelligence model for ballistic performance of thin plates. Mech Based Des Struct Mach. 2020 Nov 23;0(0):1-12.

20. Li D. Ballistics projectile image analysis for firearm identification. IEEE Trans Image Process Publ IEEE Signal Process Soc. 2006 Oct;15(10):285765.

21. Khakare MR, Fatangare S. Survey on Prediction of Post Mortem Interval using Artificial Intelligence in Forensic Examination. 2020;07(05):5.

22. Tu P, Book R, Liu X, Krahnstoever N, Adrian C, Williams P. Automatic Face Recognition from Skeletal Remains. In: 2007 IEEE Conference on Computer Vision and Pattern Recognition [Internet]. Minneapolis, MN, USA: IEEE; 2007 [cited 2021 Apr 22]. p. 1-7. Available from: http:// ieexplore.ieee.org/document/4270085/

23. Monahan J, Skeem JL. The evolution of violence risk assessment. CNS Spectr. 2014 Oct;19(5):41924.

24. Conroy MA, Murrie DC. Forensic Assessment of Violence Risk: A Guide for Risk Assessment and Risk Management. John Wiley \& Sons; 2008. 385 p.

25. Haynes J. "Brain reading," in I Know What You're Thinking: Brain imaging and Mental Privacy. Sarah D, G R, Sarah JL, editors. Oxf Oxf Univ Press. 2012;

26. Koutsouleris N, Borgwardt S, Meisenzahl EM, Bottlender R, Möller H-J, Riecher-Rössler A. Disease prediction in the at-risk mental state for psychosis using neuroanatomical biomarkers: results from the FePsy study - PubMed. Schizophr Bull. 2011 Nov 10;38(6):1234-46.

27. Douglas T, Pugh J, Singh I, Savulescu J, Fazel S. Risk assessment tools in criminal justice and forensic psychiatry: The need for better data. Eur Psychiatry J Assoc Eur Psychiatr. 2017 May;42:134-7.

28. Fazel S, Singh JP, Doll H, Grann M. Use of risk assessment instruments to predict violence and antisocial behaviour in 73 samples involving 24 827 people: systematic review and meta-analysis. BMJ. 2012 Jul 24;345(jul24 2):e4692-e4692.

29. Tortora L, Meynen G, Bijlsma J, Tronci E, Ferracuti S. Neuroprediction and A.I. in Forensic Psychiatry and Criminal Justice: A Neurolaw Perspective. Front Psychol. 2020 Mar 17;11:220.

30. Iozzino L, Ferrari C, Large M, Nielssen O, de Girolamo G. Prevalence and Risk Factors of Violence by Psychiatric Acute Inpatients: A Systematic Review and Meta-Analysis. Li S, editor. PLOS ONE. 2015 Jun 10;10(6):e0128536.

31. In Screening for Suicide Risk, Facebook Takes On Tricky Public Health Role « The New York Times in Education [Internet]. [cited 2021 Apr 22]. Available from: https://nytimesineducation.com/ 
in-screening-for-suicide-risk-facebook-takes-ontricky-public-health-role/

32. Tambekar A. How Artificial Intelligence AI can Help the Physically Challenged - Great Learning [Internet]. GreatLearning Blog: Free Resources what Matters to shape your Career! 2019 [cited 2021 Apr 22]. Available from: https:// www.mygreatlearning.com/blog/how-artificialintelligence-ai-can-help-the-physically-challenged/

33. Üstün T, Kostanjsek N, Chatterji S, Rehm J, editors. Measuring Health and Disability Manual for WHO Disability Assessment Schedule : WHODAS 2.0 [Internet]. Malta: (C) World Health Organization 2010; 2010 [cited 2021 Apr 22]. 152 p. Available from: https://www.who.int/standards/ classifications/international-classification-offunctioning-disability-and-health
34. Polyakov VV, Bespechniy OV, Neymark MA. Artificial intelligence as an object of forensic study: perspectives from a border region. In: Proceedings of the International Conference on Sustainable Development of Cross-Border Regions: Economic, Social and Security Challenges (ICSDCBR 2019) [Internet]. Barnaul, Russia: Atlantis Press; 2019 [cited 2021 Apr 6]. Available from: https://www. atlantis-press.com/article/125922364

35. Gupta S, Sharma MV, Johri DP. ARTIFICIAL INTELLIGENCE IN FORENSIC SCIENCE. 2020;07(05):4.

36. Ng WY, Norden J. Artificial Intelligence and Machine Learning. :12. 